\title{
Living with Parkinson's disease and connected to the duodopa pump: A qualitative study
}

\author{
Yolanda María Chacón Gámez, Nikola Biller Andorno \\ Institute of Medical Bioethics and History of Medicine, University of Zurich, Switzerland
}

\begin{abstract}
Parkinson's Disease (PD) is a complex and heterogeneous disease that has a major impact on the lives of patients and their family caregivers. The duodopa pump is one of the treatments options for advanced PD. The experience of patients with this treatment is largely unexplored, however. This study is based on the experiences of patients with PD as well as their family caregivers using the pump. We take a multimodal approach that includes narrative semi-structured interviews and drawings. This methodology helps participants, particularly those with difficulty articulating themselves, express their experience with the disease and their perception of the treatment. We believe that the use of drawings complementing semi-structured interviews offers additional insights into the participants' perception of PD and the pump. In this article we focus on the following topics: the participants' perception of PD and the pump, the reasons for choosing the pump over alternatives, and the advantages and disadvantages of this treatment for patients and family caregivers. Our findings show that the participants consider PD as a complex and unpredictable disease that changed their lives and affect everyone differently, while the pump makes the disease less changeable and more manageable.
\end{abstract}

\section{Introduction}

An estimated four million people worldwide suffer from Parkinson's Disease (PD), making it the second most

Correspondence: Yolanda María Chacón Gámez, Institute of Medical Bioethics and History of Medicine, University of Zurich, Wintherthurerstrasse 30, 8006, Zurich, Switzerland.

E-mail: yolanda.chacon@ibme.uzh.ch

Key words: Parkinson's disease; duodopa pump; patients' perception; family caregivers' perception; drawings.

Acknowledgements: We would like to thank to the Spanish foundation "La Caixa" for their economic contribution to our study; to Prof. Dr. Stephan Bohlhalter, Dr. Florian Brugger, Dr. Stefan Hägele-Link, and the self-help groups of the Association Parkinson Schweiz for their cooperation in our study and their help in the selection of participants; and to Dr. Matthew Baker for his assistance and comments that greatly improved this manuscript.

Contributions: YCG: Set-up of the study, data collection and analysis; literature review; manuscript write-up; manuscript editing; NBA: Research supervision; manuscript write-up; manuscript editing.

Conflict of interest: The authors declare no conflict of interest.

Funding: The Spanish foundation «La Caixa» has funded part of the study from which the data presented in this paper are drawn.

Received for publication: 30 October 2020

Accepted for publication: 7 February 2021

This work is licensed under a Creative Commons Attribution NonCommercial 4.0 License (CC BY-NC 4.0).

${ }^{\circ}$ Copyright: the Author(s), 2020

Licensee PAGEPress, Italy

Qualitative Research in Medicine \& Healthcare 2020; 4:95-106

doi:10.4081/qrmh.2020.9427 common progressive neurodegenerative disorder after Alzheimer's disease. PD is manifested by motor symptoms such as tremor, involuntary movements (dyskinesia), postural instability, rigidity, freezing episodes or slowness of movement, and nonmotor symptoms like anosmia, dementia, gastrointestinal disturbances, abnormal urinary function, hallucinations, depression, anxiety, decreased libido, apathy, sleep disturbances or difficulties swallowing. ${ }^{1,2}$ All these symptoms have a large impact on PD patients' physical and mental health, which can lead to loss of autonomy and self-esteem, altered relationships, and social isolation. ${ }^{3}$ Furthermore, PD does not present a challenge to patients only but also to their family caregivers. ${ }^{4}$ Nowadays, PD can be managed through a variety of oral pharmacological treatments such as Levodopa, dopamine agonists, transferase inhibitors or anticholinergic medication. ${ }^{1}$ Device-aided therapies such as Deep Brain Stimulation (DBS) or medicine pumps such as the duodopa or the apomorphine pump can be an option for PD patients who are refractory to oral treatment. Unlike oral therapies, infusion medicine therapies are based on constant drug delivery, which can reduce the burden of motor complications associated with PD. Currently, different options are available for medicine pumps like the continuous subcutaneous apomorphine infusion, the intrajejunal Trigel infusion or the intestinal gel infusion of Levodopa/Carbidopa, also known as the duodopa pump. ${ }^{5}$

This paper focuses on the duodopa pump (from now on the pump), which delivers levodopa/carbidopa-gel suspension directly in the duodenum through a plastic cassette linked to a pump that is connected to a tube inserted to the intestine through a small cut in the gut wall. ${ }^{6}$ This allows more stable plasma concentration of the drug than oral treatments, which improves patients' quality of life. 
In 2016, a quantitative study about the preferences of PD disease patients for device-aid treatments was conducted with 401 patients in US. Its aim was to evaluate patients' willingness to accept trade-offs among efficacy, tolerability, and convenience of alternative treatments. It showed that treatment delivered via portable infusion pump was preferred over DBS. ${ }^{7}$ However, there are many aspects of the user experience with medication pumps that we still do not know. To our knowledge, no study has been conducted that addresses patients' lived experiences with medicine pumps for PD. Thus, the personal impact of these treatments on patients' daily life is completely unexplored. We believe that we need to better understand how patients perceive medicine pumps and how they personally cope with PD symptoms and the treatment with these devices. To do this, it is also relevant to incorporate the perspective of family caregivers as a valuable source of knowledge because they are sometimes more sensitive to changes in the patient than patients themselves. ${ }^{8}$ For these reasons, we consider it very important from both a clinical and an ethical point of view to conduct more qualitative research to gain a richer understanding of patients' and caregivers' experiences, which will facilitate the continuous improvement of patient-centred care for PD patients. Our research fills this research gap by analysing the accounts of the experiences of a sample of PD patients treated with the pump and their caregivers.

\section{Materials and Methods}

The data presented in this study are part of a more extensive qualitative study conducted in Switzerland between 2018 and 2020, where we collected and explored a wide range of experiences of 44 patients with PD and aiddevice treatments (DBS or the pump) and their caregivers. We conducted multimodal research including narrative semi-structured interviews and drawings. We identified four groups of experiences reported by patients and their caregivers: i) daily life and perception of PD and aid-device treatments; ii) self-perception; iii) social interaction and partnership/family dynamics; iv) health needs of PD patients and interaction with the healthcare professionals. Due to the vast amount of data obtained during this study, in this article we will focus on the first category, which covers the perception of patients and caregivers of PD and the pump, the reasons for choosing the pump over other alternatives, and the advantages and disadvantages of this treatment for patients and caregivers. For better dissemination, our results will be displayed on the website of the Swiss Database of Patient Experiences (www.dipex.ch) over the course of 2021. Our research group is part of the international Database of Patients Experiences research initiative (DIPEx International), which comprises fourteen countries. DIPEx represents a wide spectrum of diverse perspectives about the same topic (including deviant cases) and is addressed to a wide public (patients, caregivers, healthcare professionals and students). ${ }^{8-10}$

The aim of the DIPEx website is fourfold: i) empowering patients and family caregivers to talk about their personal health experiences and to give first-hand information that health professionals not always have; ii) offering other patients and family caregiver emotional support and help with decision-making by offering them the possibility of knowing about the experiences of others in the same situation; iii) creating social awareness about how life suffering from a disease is; and iv) serving as training and educational tool for health sciences students and professionals.

\section{Study population}

A total of ten participants, including six patients treated (three men and three women) and four spouses (three women and one man) participated in the study section on experiences with PD and the pump. The characteristics of patients and caregivers are described in Table 1. Two of the patients lived in a nursing home and took part in the study alone. One of the participants was treated simultaneously with the pump and DBS. For the selection of participants, purposeful maximum variation sampling was performed to identify those individuals whose experiences were espe-cially informative and would vary from each other as much as possible.

The following inclusion criteria were applied: i) patients diagnosed with PD or caregivers providing care to a PD patient; ii) patients treated with the pump for at least six months or caregivers providing care to PD patients treated with the pump for at least six months. The following exclusion criteria were applied: i) lack of legal competency; ii) people experiencing moderate or severe dementia or substance addiction at the moment of the interview; iii) lack of physical and psychological resilience to participate in an interview or difficulties interacting with an interviewer.

The sample was small because only a low number of

Table 1. Description of patients and caregivers of the interview and details of their interviews. All participants were interviewed in 2019 except for the last participant, who was interviewed in 2020.

\begin{tabular}{lccccc}
\hline Group & Number & Average ageat interview & Average years with PD & Average yearswith the pump & Average interview duration \\
\hline Patients & 6 & 73 & 15.6 & 4.1 \\
\hline Caregivers & 4 & 70.7 & - & 91 & -
\end{tabular}


patients in Switzerland are treated with this therapy. Despite the small sample, we reached data saturation as we did not observe new themes when analysing the last interviews. We recruited the participants with the help of neurologists, PD nurses, and patient support groups belonging to the Swiss Association Parkinson's Switzerland. We conducted this study in collaboration with the Departments of Neurology of the Cantonal Hospitals in St Gallen and Lucerne.

\section{Informed consent process}

The participants were informed in great detail about the study before participating. The day of the interview, they signed the informed consent form that allowed us to proceed with the interview and its subsequent analysis for our research project. As part of the form, study participants were also given the opportunity to express their preference between being video- or audiotaped to participate. After the interview, participants received a second informed consent form, through which they could accept or reject the use of the interview for the DIPEx website and choose how they wanted the information to be presented on the website (video, audio or test). To this aim, the participants received a copy of the interview transcripts as well for verification.

Although no personal information (such as personal names or locations) will appear on the website that could lead to the identification of participants, those who have chosen to have their interview used for the website in audio or video format are aware that they may be recognised through their image or voice by people who may be able to identify them. It is therefore important that the participants had the opportunity of verifying the transcripts to decide what they would and would not like to be public information. Given that after the transcript review, not all participants may wish that some parts of their interviews would be made public, the final number of participants being included in the DIPEx platform could be lower than the number of participants in the study.

\section{Data collection}

We applied a multimodal approach that includes the conduction of narrative semi-structured interviews and the collection of drawings. This multimodal methodology allows to collect data about the experience with the disease and the treatment incorporating both language-based and nonverbal communication, which helps participants, particularly those with difficulties to articulate themselves, to express their individual experiences and give them meaning. ${ }^{11,12}$ We collected the data of the presented dataset collecting drawings and conducting ten narrative semistructured interviews in the language the interviewees felt most comfortable with. Seven were in German (with some parts in Swiss German), two in Italian and one in English and the first author conducted all of them. The first author is fluent in the three languages used in the study (German, Italian and English) and conducted all the interviews without the language being a barrier between the interviewer and the interviewee. Each interviewee was interviewed separately, but some of them were in company of the spouse and others were alone, depending on how they felt most comfortable. The interviews took place either at the participants' homes or in the nurse homes where they live.

To conduct the semi-structured interviews, we employed the DIPEx methodology, which is an established qualitative methodology developed by the research group of the department of Public Health of the University of Oxford. ${ }^{9}$ Therefore, all interviews started with a general question to give the participants the freedom to put their personal narrative into words. This question is as follows: "Could you explain your experience with Parkinson's and the duodopa pump from the beginning? How did everything start?". Once the participants had finished telling their stories, we asked a series of semi-structured questions that we separated into two blocks. The first one included questions about the participants' experiences with PD and with previous treatments like oral medication, their daily life with $\mathrm{PD}$ as patients or caregivers and their perception of PD as disease including questions about their drawing of the disease. The second section included questions about, their daily life with the pump, their perception of the pump including questions about their drawing if the treatment, the advantages and disadvantages of the pup over previous treatments as well as their reasons to choose the pump over other treatments. Additional aspects (e.g. self-perception, relationships...) were addressed during the interviews, but as it was mentioned in the introduction, they will not be presented in this paper.

To enrich the participants' oral account, we used drawings in this study as complementary qualitative methodology for several reasons. First, to draw allows the participants to visually show the consequences of chronic health conditions and share the emotions that their condition provoke on them. ${ }^{13-15}$ Second, the drawings offer additional insights to the researcher into the patients' and caregivers' experiences with PD and the pump because they provide access to nonverbal meanings and to a qualitatively different aspect of the participants' experiences. ${ }^{16}$ Third, we believe that a multimodal approach is an effective way to work with vulnerable participants and address sensitive topics. ${ }^{17}$

At the beginning of each interview, both patients and caregivers were invited to draw how they visually perceived the disease and the pump. Although other authors usually ask the participants to draw after the interview, ${ }^{18}$ we decided to ask our participants to draw before conducting the interview to provide our participants with the opportunity to reflect on their own history and circumstances before recounting it, because we considered that this preliminary reflection would contribute to enriching the results obtained from the study. The participants re- 
ceived a sketching pencil, a set of twelve colour pencils, an eraser and two pieces of paper (one to draw their visual representation of PD and another for the pump). Five participants opted not to draw due to physical incapability or the lack of a visual image of the disease or therapy. During the interview, the participants who drew were asked different questions about the drawings they had made. It was not necessary for the participants to know how to draw well. It only mattered that their drawing had a meaning for them that they could later explain during the interview.

\section{Data analysis}

We performed our data analysis using a modified approach of grounded theory, which required a continuous back and forth between data collection and analysis. ${ }^{9,19} \mathrm{To}$ proceed with the analysis, the interviews were transcribed verbatim by our team of transcribers. After the participants' verification, we fed the transcripts and the drawings into the qualitative data analysis software MAXQDA, which allowed us to perform multimodal analysis. Afterwards, we thematically coded data using a coding scheme (coding tree), which we primarily created based on the interview guide and continuously enriched with topics emerging from the transcripts and the drawings (mix coding). ${ }^{20}$ Every time that we created a new category or code, we reviewed all coded segments to ensure homogeneity within the entire data set. This way, the concepts that we used to develop the final coding tree stem from the collected participants' life experiences through the semistructured interviews and the drawings, which we subsequently systematized, categorized and analysed following the coding tree. ${ }^{9}$ The final coding tree was checked by other members of our team to ensure trustworthiness. The coding tree was created in English to allow the discussion about it with other members of the team who do not speak German or Italian, but all data were coded and analysed in its original language by the first author. The second author supervised the analysis.

Further analysis will select relevant material for our website www.dipex.ch using the One Sheet Of Paper (OSOP) method, which involves reading through each section of coded data for each topic and summarize on a single sheet of paper the key points of all interviews in relation to the same topic. ${ }^{9}$ The resulting text will constitute the summary of the different topics for the website. The website will be organized in the categories derived from the coding tree (e.g. PD symptoms, patients' or caregivers' difficulties with PD, work life with PD...), and all texts, video and audio clips on the website will be classified along these categories. ${ }^{9} 10$

\section{Ethical concerns and data management}

The study participants were not at risk of any physical harm and did not directly benefit from the study. We conducted this study in compliance with the current version of the Declaration of Helsinki, the ICH-GCP and ISO EN 14155 (as far as applicable) as well as all national legal and regulatory requirements. The study has been reviewed by the ethics review committee of the Canton of Zurich, which considered that it did not fall under the Swiss Law on Human Subjects Research and, after consultation with the national working group of Swiss ethics review committees, issued a nation-wide waiver. Subsequently, our study proposal went through our Institute's internal ethics review process (CEBES). We handled all data confidentially and only persons who are directly involved in the data collection, transcription or data analysis had access to them. Data are protected by copyright and belong to the Institute (the name will be provided when the manuscript is accepted for publication), which can use the collected data for research and teaching purposes including publications or broadcasting.

We performed data anonymization and we saved the data of each participant giving them a code (e.g. Patient1, Caregiver1...) and deleting from the transcripts any information that that could lead to the personal identification of participants. In order to present our results in this paper, we translated the selected quotations of the interviews that were not conducted in English using the DeepL Pro Translator. The translated data were not stored by DeepL and all translations were compliant with our data protection regulations. The final translations were verified by a native speaker to ensure accurate translation of the original quotes.

\section{Results}

\section{Patients' and caregivers' perception of PD}

The reactions of the different participants when receiving the diagnosis were very different from each other. While most participants, both among patients and caregivers, stated that they were not surprised by the diagnosis of PD, one participant stated that she felt devastated when she received the $\mathrm{PD}$ diagnosis:

\footnotetext{
“That was a shock to me because I didn't know how it would play out, how it would progress. The diagnosis blows your mind. It smashes you and you feel disappointed. The truth is difficult to accept. The diagnosis is difficult to accept (...)." (patient 6).
}

"What was the most difficult thing for you to accept?" (author 1)

\section{"That it is incurable." (patient 6).}

Some of the participants even expressed relief at the diagnosis because they were aware that something was wrong and did not know what. Others admitted not being aware of what it meant to have PD. One of the patients said the following: 
"I had no idea that it affected the whole body. I thought I just end up with tremor one day. I had no idea what it meant. And a lot of people don't know what it means. Even medical people (...) I was ignorant. I didn't know what was ahead of me. So, they probably thought I was just cheerful by nature, but I was ignorant (...)."

So, you didn't feel shocked? (autor 1)

No. I just carried on. You know and then slowly or suddenly I couldn't do things." (Patient 4).

This feeling of discovering that living with PD was much harder than they expected at the time of diagnosis was shared by other participants. When explaining their experiences with the disease, different participants expressed things such as: "This is a terrible disease" (caregiver 3); "It's just a break in life" (caregiver 1) ;"I believe that I have been a victim of this disease for a long time" (Patient 1) or "Every day I also see that there is a change in the worse possible sense" (Patient 5). One of the caregivers explained that his wife went through a very difficult period with the disease two years ago when she considered applying for assisted suicide (which is legally allowed in Switzerland). Despite the difficulty of living with this disease chronically either as a patient or as a family caregiver, most expressed relief that PD is not a fatal disease. For instance, one of the patients, who also suffered from cancer, said:

"Parkinson's makes you sick, but you don't die"

(...) Cancer was worse than Parkinson's because it was eating me." (Patient 5).

Another experience shared by virtually all patients and caregivers is the impression that PD changes your life but not in the same way for everyone because everybody's PD is different. Not only can each person have different symptoms, but each patient faces them differently, which means that each of them goes through the disease in a different way. While some responded to PD symptoms in a more physical way by trying to be active most of the day as they experienced less symptoms if they were focused on some activity, others dealt with the symptoms rather in a mental way by trying to think positively about the disease. One of the patients mentioned that facing PD with a sense of humour is the most important thing for her to not feel swamped by the disease and described how to find the fun side of the disease.

"I can't live without a sense of humour/ you can't live without. You've got to see the other side as well (...) My mother had a very good sense of humour and was always giggling. So, maybe I grew up with that mentality (...) But since I have Parkinson's, I laugh much more. When I come together with friends and I tell them these stories that happened to me, and they're very serious stories. We should really cry, but we just end up laughing (...). In rehabilitation, another woman who had deep brain stimulation and we were always laughing at one another, you know, because uhm/ these/ all these hitches, all things that didn't work (...) And we had so much fun. (...) If you don't get drowned by the situation, it's very funny." (patient 4).

If we look at the way some patients and caregivers visually captured the disease on paper through their drawings, we get more extra information about how they perceive the disease.

In Figure 1 we can see how patient 4 visualises her feeling that her motor skills fluctuate during the day through the use of different colours. She represented PD symptoms as a figure in blue, and the effect of the oral medication consisting of levodopa as different figures in red, orange, and yellow. Although this treatment improved her motor skills, its effect was not stable because it was wearing off and she began to experience off-periods. Therefore, she represented movement through various figures that are intermingled during the day with the PD symptoms.

"Well, the colours for me, the red, orange are like dynamo, like movement and dynamics. And the blue is the more rigid, is a rigid position, a rigid / the rigid leg. That's how I depict Parkinson's, rigidity." (patient 4 ).

Rigidity is for you the definition of Parkinson's? (first author).

Yeah. Stiffness (patient 4) (...)

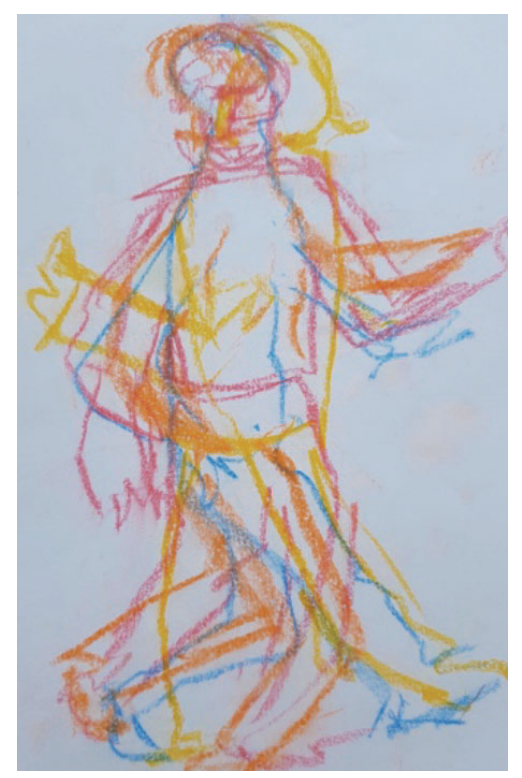

Figure 1. Patient's 4 perception of PD. 
So, the blue figure is the underlying disease? (first author).

Yeah, yeah. And that's the release with something like levodopa. These colours there are the movement (pointing at the figures in red, orange and yellow)." (patient 4).

The unpredictability of PD is reflected, in two different ways, in Figure 2 from the perspective of one the care-givers.

On the one hand, as PD is degenerative disease, there is always the big question of how the disease will develop in the long term and how life will be then, which is unpredictable. On the other hand, patients and caregivers need to cope with the unpredictability of the daily fluctuations of PD symptoms, which makes daily life difficult.

"That's the Parkinson's. That is, yes, exactly, the Parkinson's. You don't know what to do next. (....). And here, what's difficult with Parkinson's is the fluctuations (pointing at the curve) (caregiver 3). And what does the straight-line mean? (first author). This is a medicine. Different drugs. (...) I don't know where the limit is that the medication stops working. Doctors don't know that either. With one person it takes longer to reach it and with others less. We need to live every day." (caregiver 3 ).

\section{Patients' and caregivers' perception of the pump}

In Figure 3, we see how patient 4 perceives the effects of the pump differently in comparison to oral treatment.

While in Figure 1 we could observe several figures in different colours reflecting movement, which contrasted with the blue figure representing PD, in Figure 3 we can see how patient 4 perceives the effect of PD as a single orange figure that is jumping and contrasts with the rigidity of the figure in blue. This representation reflects her perception of the effect of the pump being more stable and consistent than the effect of oral medication, which gives her greater motor mobility throughout the day.

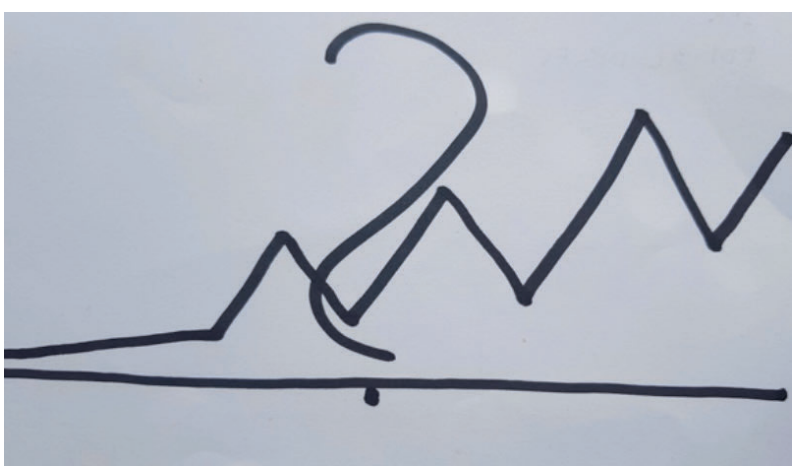

Figure 2. Caregiver's 3 perception of PD.
"This is a stooping figure (pointing at the blue figure). That's what Parkinson's does to most people. But this is what I feel like when I feel when I fed/ fed the pump (pointing at the orange figure). You know I feel much livelier. Everything goes upwards instead of downwards. That's a mix of/that's what I feel like with the pump, okay? (...) Just, you know, not dragged downwards, but moving upwards. I can move more easily and uhm I can walk very quickly when I'm/when I'm feeling good." (patient 4).

Although she was very satisfied with the pump as treatment, she described a sense of dependence on the pump to be able to move properly.

"I'm now living with uhm uhm an attachment to me (...) Now I'm not dependent on tablets, but I'm dependent on the cassette, am I not?"

Looking at the caregivers' perception of the treatment, one of them compares their experience with the pump in comparison to oral medication in Figure 4.

"Those are the drugs (pointing at the circles on the top of the drawing, which represent the hours his wife had to take her pills every day). She had to take 8 pills a day (...). Every day she was suffering from Parkinson's disease from morning to night, because she was constantly reminded that she has Parkinson's disease. And with the pump we turn it on and off." (caregiver 3).

The pills taking routine was replaced by the pump cleaning, activation and deactivation routine, which is carried out by the patient's husband. He explained that each

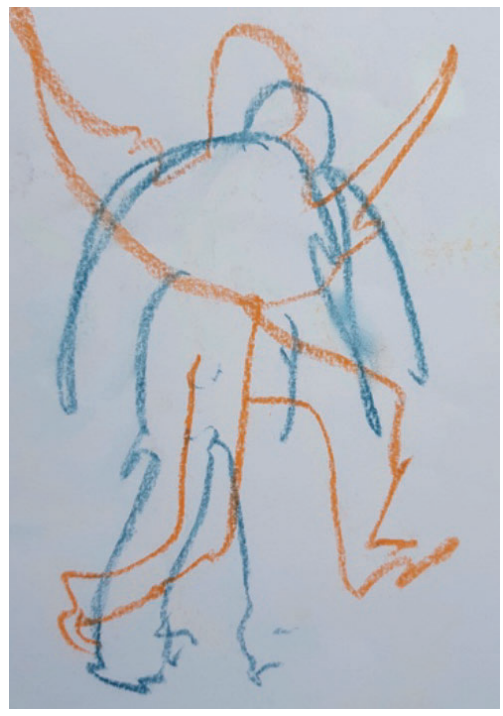

Figure 3. Patient's 4 perception of the pump. 
morning at 06.00 , he takes the levodopa/carbidopa-gel out of the fridge to let it warm up a bit before putting it in the cassette of his wife's pump at 08.00 to switch on the pump on for the day. In the evening, he switches the pump off at 18.00 , and at 20.00 he cleans the tube that goes into the patient's intestine. While caregiver 3 was explaining his drawing, he mentioned that he started a diary since his wife was treated with the pump. He used it to keep track of the involuntary movements that his wife suffers throughout the day to accordingly adjust the dose of medication put into the cassette.

"The pump is much simpler. But the adjustment is very, very difficult (...) An adjustment can be good for three or four days and suddenly it's nothing. There must be other events and then there can be fluctuations. But I think it's much less today than it was before with the medication (...) I take some minutes to write down every day the symptoms and the duration because you can't keep everything in your head. Mr. (Name_1) is our doctor and gave us free hand in varying the dosage. He gave us some recommendations and said, "try it once". I am also aware that every Parkinson is different. Every person reacts differently. This is very difficult. But that's what it is with these drugs."

\section{The reasons to choose the pump over other treatments}

Most participants chose the pump as a treatment because oral treatment was no longer effective for management of their PD symptoms and their quality of life was very poor. Most of them expected to enjoy greater mobility with the pump and noted that the PD symptoms decreased with this treatment (e.g. less uncontrolled movements or freezing episodes). For one of the patients, the reason to choose the pump over oral treatment was that the side-effects of drugs taken orally were unbearable. His wife explained that the treatment with dopamine agonists caused him to have, as a side effect, a serious sex addiction, which led him to spend a great amount of money in sexual relations with several prostitutes.

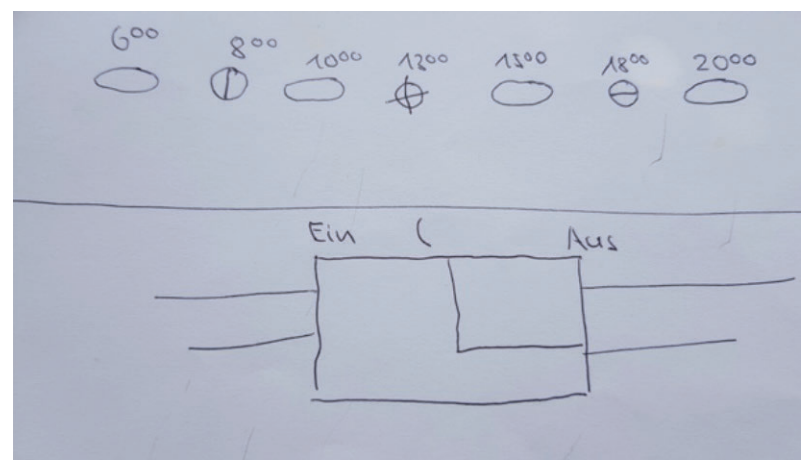

Figure 4. Caregiver's 3 perception of the pump.
"He took drugs, various drugs. At first, they fluctuated and also people change. He was not the same person anymore, because with the pills, it always goes high and then it goes down again and then up again (...) He got stronger and stronger medication over the years (...) And the pills have very bad side effects. Especially the Sifrol that was what he got. And then many unpleasant things happened. So violently. And that was the reason why/I immediately told the doctor (...) He cheated on me for years with prostitutes. That was a disaster (...). That's why doctor (name_1) said that with the pump would be much better." (caregiver 1).

Two patients were offered the possibility to choose DBS. One of them was doubtful at first but ended up rejecting it because she would not have felt comfortable with a device inside her brain. Her husband explained that he did not know how he would have reacted if his wife would have chosen DBS instead the pump because DBS is a treatment he could not morally agree with.

"At first she was not determined. For her it was an opportunity. And for this reason, we were informed at the hospital. How much that I influenced her in her decision, I do not know of course. But for me it was never an issue. This is a catastrophe for me. (...) Drilling holes in the brain, that's not possible. A person is not a machine where you can screw in a screw. For me there are limits in all medicine. And it borders on ethics (caregiver 3).

So deep stimulation is not an ethical treatment for you? (first author).

No, no, this is a mechanical treatment (caregiver 3). Why is DBS not ethical?

I am not a religious person, not at all, but I think that many things are wanted, somewhere wanted it must be so. And we have to learn to accept a disease, not to accept, but to come to terms with it. That is also a difference for me. Accepting it means doing nothing else, isn't it? But coming to terms with it means that we simply cannot expect to be able to correct everything, everything. (...) From my point of view, it is irresponsible (caregiver 3).

What is irresponsible? (first author).

Drill holes there. That might take millimeters, millimeters. Millimeters and then it happened. And then I would have a woman who couldn't do anything anymore, maybe. The risk was also much too big for me." (caregiver 3).

Another patient, who was not offered DBS, nonetheless was of the same the opinion as caregiver 3 . He mentioned that if he had been offered DBS as a therapeutic option for him, he would have not accepted anyway because of ethical reasons. 
"The brain stimulation was out of question for me also for ethical reasons. I find it delicate when the skull is sawed open." (patient 1).

The other patient who was offered DBS as treatment did not consider the option of being treated with it because she also was too afraid of having a hole in her skull.

"I find it grotesque; I mean having a whole board in your head. And it doesn't last, it doesn't, it doesn't make you immune from the results (...). Is it worth it?" (patient 4).

(...) Would have been stressful or disturbing for you to be treated with it though? (first author).

(...) I can't imagine what it's like having a hole in your head. And they shave away your hair and you don't know whether it's going to be a success (...) It's very invasive, isn't it? (...) I think it's incredible people are prepared to do that. (...) Would you do it? (patient 4).

The four couples who participated in the study explained that they assessed all the options together and jointly made the decision to try with the pump. The three caregivers felt very involved in the decision-making process, and all three patients felt supported by their caregivers. All participants (including the two patients who participated in the study without the participation of a caregiver) expressed satisfaction with the decision taken. Several of them explained that a major reason for deciding on the pump was that it was an easy treatment to stop if it did not work well, whereas stopping DBS would have been more complicated. In addition, before any perforation was made in the wall of the duodenum, the effectiveness of the treatment was tested by inserting a tube through the patient's mouth temporarily for a few days. This was evaluated positively by patients and caregivers, although one patient felt discomfort after one day and asked for the definitive tube to be inserted through the duodenum.

\section{Advantages and disadvantages of the pump}

All participants expressed having noticed a marked improvement when they started being treated with the pump and a better quality of life. One patient was being treated at the same time with DBS and the pump and it was planned to remove the pump when DBS would start to work properly for the patient. At the time of the interview DBS was not controlling the PD symptoms to the level expected by the doctors. The patient was also more satisfied with the improvement achieved with the pump than with DBS, although he acknowledged that a proper management of the PD symptoms was lately also being difficult with the pump as well. Despite this, he considers the pump as a good transition between the pills and DBS, because he could not imagine going from oral treatment straight into DBS. Some of the advantages mentioned by participants were better sleep (without waking up more than once or twice during the night), a better management of PD symptoms than with pills and feeling better during the day. The relief of not having to take pills every three or four hours was shared by both patients and caregivers, as the daily routine revolved around the time the pills needed to be taken. One of the caregivers explained that her husband could eat better now that he didn't have to take so many pills a day.

Two of the caregivers mentioned that the pump calmed their spouses greatly and they were more psychologically stable than when their treatment consisted of oral medication. In one of the cases, the reason for the increased stability was that her husband no longer suffered from sexual addiction caused by oral medication and did not seek sex with prostitutes, which markedly improved the relationship between them. In the other case, the family caregiver explained that her husband had great fluctuations in his mood and suffered from deep depression when he was on oral medication. Once the patient received treatment with the pump, he experienced no ups and downs.

However, participants also reported some disadvantages they found with the pump as therapy. For example, one caregiver mentioned some disadvantages that were shared by other participants:

"You are simply not that free or flexible anymore (...) Yeah, it's just that you're just sort of fixated. In theory one could do anything, but I find it too delicate. An example is swimming. One can go and cover it with special plasters. The people in charge of the pump say of course, it's no problem, you can do it. But I am too sceptical. Nowadays in these swimming pools, or, with all these chemicals and chlorine, you don't know what can happen, so I just don't trust it. That's the puncture that goes in, and what if some bacteria get in there. So, you're just restricted with sports (...) And then with yoga one can't do some things anymore because otherwise you lie on the pump, don't you? One can still do exercises, but not many. If the pump were smaller, that would be better. And also, with travelling, you always have to take everything with you. I always have medicine in my handbag, if the pump breaks down, or if he needs an extra dosage. Then I also must always have batteries with me in case the pump runs out of battery." (caregiver 1)

The impossibility of pursuing some activities with the pump, that patients pursued before starting treatment, was described by other participants. For example, patient 5 used to exercise daily with a stationary bike and had to stop doing it when she started to be treated with the pump. Patient 2 mentioned that he had to stop swimming, as previously mentioned by caregiver 1 , although he was also told 
that swimming was possible after removing the pump and tube. However, his reason for stopping swimming was not due the fear to bacterial infections but to the concern that during the time he did not have the pump, he would have PD symptoms that he could not control otherwise.

The large size and weight of the pump was also an aspect regarded as an inconvenience by most of the patients. One of the patients described having to carry such as a large device as feeling like being a kangaroo with a pouch. The pump size and weight were also reasons why some participants had to stop doing certain physical activities like riding a stationary bike or doing yoga. Since doing physical activity is considered by patients to be essential in coping with $\mathrm{PD}$, it poses a problem for them not to be able to do physical activities that they previously did because of the size of the pump. Patient 1 related the large size of the pump to a lack of technological development of the pump by the industry. He considers that the companies that make this pump should pay more attention to the daily needs of PD patients.

"If you already have such a disease, then you should make your day as easy as possible for the patients and then you are abandoned by the industry." (patient 1).

Only one of the patients explained that for him the pump size is not a problem as over time one gets used to it and does not notice it anymore. Finally, diverse accounts regarding the care of the pump were shared. While one caregiver reported that the daily cleaning of the tube connecting the patient's duodenum to the pump was very laborious and time-consuming, another caregiver stated that this task was very bearable for him and was not a burden in his daily life. The two other caregivers explained that they did not take care of the cleaning of the tube because their husbands could still take care of it. In the case of the two women living in a nursing home, the tube cleaning was assumed by the nurses working in the centre.

\section{Discussion}

PD is a very heterogeneous disease not only in the symptoms it can present but also in the progression of the disease over time. ${ }^{21}$ This heterogeneity can cause problems in diagnosing the disease and also in finding the right treatment for each patient. As PD is a chronic and degenerative disease, patients try different medical treatments throughout their lives. From a pharmacological point of view, there are a wide variety of oral treatments to manage PD (e.g. Levodopa, dopamine agonist, transferase inhibitors, anticholinergic medication). ${ }^{1}$ However, pharmacological management of PD can be especially challenging when motor complications can no longer be controlled by changes in oral treatment or by combinations of drugs. Worsening symptoms due to disease pro- gression or increasing side-effects caused by some oral treatments have an undeniable impact on the quality of life of patients and their families, as the life and daily routines of caregivers is also affected in such situations. ${ }^{22,23}$ Different aid-device therapies such as DBS or medicine pumps are available if conventional pharmacological treatments fail. These therapies differ in their invasiveness, side-effect profile, and the need for nursing care and work differently in each patient. ${ }^{24}$

Our results reveal that the duodopa pump is considered by our participants as a treatment capable of minimising the signs of PD by facilitating the daily life of patients and their caregivers. Some of the described advantages of this treatment were better management of PD symptoms than with oral treatments, fewer side-effects, increased comfort due to not having to take pills all the time, improved quality of sleep, and greater psychological stability. All this translates into an improved quality of life of patients and caregivers. Nevertheless, some discomforts in daily life with the pump have also been described: e.g. difficulties in practicing sports like swimming or yoga, the need of greater advanced planning when travelling, the discomfort due to the great weight and size of the device, the time consuming task of cleaning the tube connected to the intestine or the feeling of being tied to a device. Nonetheless all participants were satisfied with the choice of being treated with the pump, reducing the size and weight of the device could help to alleviate the above-mentioned discomforts and further improve the quality of life of patients and their caregivers. Furthermore, our results show that the pump could be a therapeutic alternative for those people who are reluctant to undergo DBS because of insurmountable fear, due to ethical reasons or who would not feel comfortable with a device deep in their brains for other reasons.

Our study also provides relevant information about the participants' perception of PD as a disease that elicits heterogeneous reactions both in the acceptance of the diagnosis and in coping with the disease in their everyday life. ${ }^{25-27}$ While some patients received the diagnosis with relief or were not impacted by the diagnosis of PD due to lack of knowledge about the disease and not knowing exactly what they were facing, one patient described the diagnosis as devastating and difficult to accept. What all participants seem to agree on is that PD ended up being a very difficult disease to deal with, which is different for everyone. Therefore, each patient deals with the disease in a different way by establishing different coping strategies (e.g. by being active, facing the disease with sense of humour, or thinking positively). The caregivers also need to establish their own coping strategies (e.g. establishing mechanisms like a diary to understand how PD symptoms develop over the course of a day for their loved ones ), because they play a fundamental role in the daily management of the disease. ${ }^{28-30}$

Our results also show that PD is accounted for as a 
less bad disease to cope with than other diseases, such as cancer, because it is not considered a terminal disease. This finding seems to contrast with the perception of other chronic patients, who consider that the consequences of Chron's disease are similarly strong as those of patients with cancer. ${ }^{31}$ However, other study about the patients' experiences with multiple sclerosis show that the diagnosis of this disease dissipates fears of acute danger, which is comparable to what our participants expressed when considering that PD is not a fatal disease. Another similarity between the perception of our participants with PD and the patients with multiple sclerosis participating in the Reenen et al. study is that the initial relief is challenged by the realization that PD is a disease that progresses and worsens over time. ${ }^{32}$ This coincides with the difficulty of our participants to cope with the disease progression and its unpredictability that characterises PD both in the manifestation of its symptoms and in its progression.

Our multimodal approach including narrative semistructured interviews and drawings allowed us to obtain an additional insight into the participants' perception of PD and the effects of the pump. The integration of drawings as a complementary methodology to the interviews enabled us to better understand the complexity of the reality that they live in dealing with their disease and its treatment. For instance, the use of curves and lines allowed caregiver 3 to represent in Figure 2 the fluctuation of their wife's symptoms during the day and the uncertainty it causes due to not knowing what will happen next. Through the use of colour, patient 4 could describe the phenomenon of wearing-off of dopaminergic drugs through different figures in yellow, red and orange tones that overlap each other in Figure 1, and in Figure 4 the contrast between the PD symptoms and the effects of the pump. The body drawn in orange that is jumping reflects her perception of the effects of the pump as continuous and unchanging, which not only makes her feel more alive but also more confident, by providing her more control over her illness than the oral medication she was taking before starting the treatment with the pump. This sense of greater control over the disease is also apparent from Figure 4 , in which caregiver 3 pictures the pump as a device that figuratively allowed his wife to turn the PD on and off.

We decided to adopt a multimodal approach to explore how different modes could make meaning in ways that are creative and that goes beyond the pre-established meaning of words. ${ }^{33,34}$ Although the use of drawings to explore the way in which people understand and experience different diseases was traditionally limited and mostly confined to the paediatric field, ${ }^{18,35}$ their use has slowly emerged in the last decade as a valuable tool complementing semi-structured interviews for investigating patients' perceptions about their illness and treatments that are difficult to verbalize. ${ }^{36}$ For instance, Scott explores the concept of AIDS among women living with HIV adopting a multimodal approach including interviews, free lists and drawings ${ }^{37}$ Besser et al., asked their participants to draw during the interviews to have a further insight into how patients view osteoporosis and its consequences in their daily lives, which elicited powerful emotions from the participants. ${ }^{38}$ Kirkham et al., in their study about experiences with chronic pain, used the drawings to explore the participants' individual representations of their pain, which they consider an invisible condition and difficult to describe with words. ${ }^{39}$ This description of the pain through the colour coincides with the representation of the motor skills fluctuations typical of PD and the effects of the pump on the improvement of its symptoms depicted by patient 4 through the drawing of body figures in different colours.

\section{Strengths and study limitations}

Our study applies the narrative DIPEx approach, which allowed us to explore in-depth the perception of patients and caregivers of PD and the management of it with the pump. To our knowledge, it is the first study exploring their personal experiences with this treatment and its impact on the patients' and caregivers' daily life. The perspective of caregivers provided us with valuable information about PD and the advantages and disadvantages of the pump that patients cannot always offer. Despite the small sample, the study has managed to collect results that reflect different types of circumstances, experiences, impressions and opinions of both patients and caregivers. Some participants, both patients and caregivers, experienced a beneficial emotional effect for having been listened to and for their personal stories having been taken into account for research. Furthermore, we believe that the use of drawings as complementary methodology to narrative semi-structured interviews elicited the further verbal data because they allowed the participants to reflect on what they have drawn and express these reflections during the interview. ${ }^{16}$ The possibility of expressing themselves through drawings also gave them the opportunity to express emotions that would not have been externalised by words alone and facilitated the communication of difficult experiences..$^{40}$ Therefore, our multimodal research including narrative semi-structured interviews and drawings offer additional insights into the participant's experiences not only with the disease but also with the treatment, which led to a more complete data analysis.

We identified three possible limitations in our study. Firstly, the participants were only interviewed after being treated with the pump and not before they started receiving this treatment. Although all participants were asked questions about their daily lives and how they were doing before treatment, those who have lived with PD and the pump for a longer period of time may have lost perspective on the before and after. Secondly, we could have missed some negative experiences with the pump due to the fact, that from our experience, people who have had bad experiences are often more reluctant to share their experiences than those who have had positive experiences with the treat- 
ment. Likewise, the perception of the pump users opting against DBS needs to be further investigated. Thirdly, our findings may be linked to a specific cultural setting, which could make it difficult to extrapolate them to other countries. It would therefore be valuable to carry out this study in other countries so that the results can be compared.

\section{Conclusions}

PD is a complex and heterogeneous disease that changes the lives of patients and their caregivers from the moment of diagnosis affecting all facets of their daily lives. ${ }^{26,29}$ From a clinical perspective, finding the right treatment for each patient is neither an easy task. Medicine pumps are one of the options along with DBS for advanced PD when oral medication stops working. However, the user experience with these medication pumps is largely unexplored. Since the patient's perception of the chosen therapy may also play a role in the final outcome ${ }^{41}$ it is important to better understand how patients, and with their caregivers, perceive medicine pumps and their experiences with these pumps. Therefore, the presented study describes the experiences of a small group of patients and caregivers with PD and the pump. The analysed experiences offer valuable insights into the lived experience of living with PD and being treated with the pump, the caregivers' perspective of this situation and the reasons that led the participants to choose the pump as therapy over other alternatives.

We conducted multimodal research using drawings and narrative semi-structured interviews with the aim of exploring the complexity of the individual experience with a chronic degenerative disease treated with a device-aid therapy. Drawing give the participants the opportunity to convey emotions through the use of colour and shape that give words a new dimension. Furthermore, multimodal research methods allow us, the researchers, to look at the patients' and caregivers' experiences from more than one perspective, which offer us the possibility of capturing the sensate aspect of the illness. Therefore, we advocate for more research done following a multimodal approach, which enables that the illness experience and the process of communicating it to another person are embodied. ${ }^{11}$

Our findings show that the participants consider PD as an unpredictable disease that changed their lives, while the pump makes the disease more manageable, which improves the life quality of patients and caregivers. Some disadvantages of the treatment have also been pointed out by the participants. Where there was most agreement was that the size and weight of the pump was excessive, limiting the ability to perform certain activities. Therefore, further technological developments leading to a reduction of the pump size and weight would be desirable.

The impression of being dependent on or tied to the device was also reported and hence, future research is needed to explore this topic further. It would also be rel- evant to carry out a comparative study between different countries to assess whether the cultural component plays a role in patients' and caregivers' perception of medication pumps. As a final conclusion, our findings show that despite the drawbacks, our participants were satisfied with the improvements that the pump has brought to the patients' physical and mental health. From the perspective of our participants, these improvements could not have been achieved otherwise.

\section{References}

1. Hayes MT. Parkinson's Disease and Parkinsonism. Am J Med 2019;132:802-7.

2. Kulisevsky J, Oliveira L, Fox SH. Update in therapeutic strategies for Parkinson's disease: Curr Opin Neurol 2018;31:439-47.

3. Soundy A, Stubbs B, Roskell C. The Experience of Parkinson's Disease: A Systematic Review and Meta-Ethnography. Sci World J 2014;2014:613592.

4. Calne SM. The Psychosocial Impact of Late-Stage Parkinson's Disease. J Neurosci Nurs 2003;35:306-13.

5. Ray Chaudhuri K, Qamar MA, Rajah T, Loehrer P, Sauerbier A, Odin P, et al. Non-oral dopaminergic therapies for Parkinson's disease: current treatments and the future. Npj Park Dis 2016;2:16023.

6. Odin P, Wolters E, Antonini A. Continuous dopaminergic stimulation achieved by duodenal levodopa infusion. Neurol Sci 2008;29:387-8.

7. Marshall T, Pugh A, Fairchild A, Hass S. Patient Preferences for Device-Aided Treatments Indicated for Advanced Parkinson Disease. Value Health 2017;20: 1383-93.

8. Hariz G-M, Hamberg K. Perceptions of Living With a Device-Based Treatment: An Account of Patients Treated With Deep Brain Stimulation for Parkinson's Disease: Parkinson Patients' Views of Their DBS Device. Neuromodulation Technol Neural Interface 2014;17:272-8.

9. Ziebland S, McPherson A. Making sense of qualitative data analysis: an introduction with illustrations from DIPEx (personal experiences of health and illness). Med Educ 2006;40:405-14.

10. Ziebland S, Herxheimer A. How patients' experiences contribute to decision making: illustrations from DIPEx (personal experiences of health and illness). J Nurs Manag 2008;16:433-9.

11. Jewitt $\mathrm{C}$, editor. The Routledge handbook of multimodal analysis. Second edition, first published in paperback. London New York: Routledge, Taylor \& Francis Group; 2017: p. 527.

12. Adami E. Multimodality. 2015. Accessed 2021 Feb 8. Available from: http://rgdoi.net/10.13140/RG.2.1.4818. 0565

13. Broadbent E, Petrie KJ, Ellis CJ, Ying J, Gamble G. A picture of health-myocardial infarction patients' drawings of their hearts and subsequent disability. J Psychosom Res 2004;57:583-7.

14. Thorpe C, Arbeau KJ, Budlong B. 'I drew the parts of my body in proportion to how much PCOS ruined them': Experiences of polycystic ovary syndrome through drawings. Health Psychol Open 2019;6:2 055102919896238.

15. Broadbent E, Niederhoffer K, Hague T, et al. Headache sufferers' drawings reflect distress, disability and illness perceptions. J Psychosom Res 2009;66:465-70. 
16. Brailas, A. Using Drawings in Qualitative Interviews: An Introduction to the Practice. Qual Rep 2020;25: 4447-60.

17. Morgan M, McInerney F, Rumbold J, Liamputtong P. Drawing the experience of chronic vaginal thrush and complementary and alternative medicine. Int $\mathrm{J}$ Soc Res Methodol 2009;12:127-46.

18. Guillemin M. Understanding illness: using drawings as a research method. Qual Health Res 2004;14:272-89.

19. Strauss AL, Corbin JM. Basics of qualitative research: techniques and procedures for developing grounded theory. 2nd ed. Thousand Oaks: Sage Publications; 1998: p. 312.

20. Bryman A. Social research methods. Fifth Edition. Oxford; New York: Oxford University Press; 2016: p. 747.

21. Greenland JC, Williams-Gray CH, Barker RA. The clinical heterogeneity of Parkinson's disease and its therapeutic implications. Eur J Neurosci 2019;49: 328-38.

22. Borovac JA. Side effects of a dopamine agonist therapy for Parkinson's disease: a mini-review of clinical pharmacology. Yale J Biol Med 2016;89:37-47.

23. Padovani C, Lopes MC de L, Higahashi IH, Pelloso SM, Paiano M, Christophoro R. Being caregiver of people with Parkinson's Disease: experienced situations. Rev Bras Enferm 2018;71:2628-34.

24. Volkmann J, Albanese A, Antonini A, Chaudhuri KR, Clarke CE, de Bie RMA, et al. Selecting deep brain stimulation or infusion therapies in advanced Parkinson's disease: an evidence-based review. J Neurol 2013;260:2701-14.

25. Merritt RK, Hotham S, Graham L, Schrag A. The subjective experience of Parkinson's disease: A qualitative study in 60 people with mild to moderate Parkinson's in 11 European countries. Eur J Pers Centered Healthcare 2018;6:447.

26. Soleimani MA, Bastani F, Negarandeh R, Greysen R. Perceptions of people living with Parkinson's disease: a qualitative study in Iran. Br J Community Nurs 2016;21:188-95.

27. Plouvier AOA, Olde Hartman TC, van Litsenburg A, et al. Being in control of Parkinson's disease: A qualitative study of community-dwelling patients' coping with changes in care. Eur J Gen Pract 2018;24:138-45.

28. McLaughlin D, Hasson F, Kernohan WG, et al. Living and coping with Parkinson's disease: perceptions of informal carers. Palliat Med 2011;25:177-82.

29. Chiong-Rivero H, Ryan GW, Flippen C, et al. Patients' and caregivers' experiences of the impact of Parkinson's disease on health status. Patient Relat Outcome Meas 2011; 2011:57-70.
30. Rastgardani T, Armstrong MJ, Marras C, Gagliardi AR. Improving patient-centred care for persons with Parkinson's: Qualitative interviews with care partners about their engagement in discussions of 'off' periods. Health Expect Int J Public Particip Health Care Health Policy 2019;22:555-64.

31. van der Have M, Minderhoud IM, Kaptein AA, et al. Substantial impact of illness perceptions on quality of life in patients with Crohn's disease. J Crohns Colitis 2013;7:e292-301.

32. Van Reenen E, Van Der Borg W, Visse M, et al. Fear, fight, familiarize: the experiences of people living with relapsingremitting multiple sclerosis and taking oral medication. Int J Qual Stud Health Well-Being. 2019;14:1648946.

33. Jewitt C. Multimodal Methods for Researching Digital Technologies. In: The SAGE Handbook of Digital Technology Research [Internet]. London, United Kingdom: SAGE Publications Ltd; 2013. Accessed 2021 Jan 29. p. 250-65. Available from: http://methods.sagepub.com/book/the-sagehandbook-of-digital-technology-research/n18.xml

34. Bock Z. Multimodality, creativity and children's meaningmaking: drawings, writings, imaginings. Stellenbosch Pap Linguist PLUS [Internet]. 2016;49:1-21.

35. Rollins JA. Tell me about it: drawing as a communication tool for children with cancer. J Pediatr Oncol Nurs Off J Assoc Pediatr Oncol Nurses 2005;22:203-21.

36. Cheung MMY, Saini B, Smith L. Using drawings to explore patients' perceptions of their illness: a scoping review. J Multidiscip Healthcare 2016;9:631-46.

37. Scott A. Illness Meanings of AIDS Among Women With HIV: Merging Immunology and Life Experience. Qual Health Res 2009;19:454-65.

38. Besser SJ, Anderson JE, Weinman J. How do osteoporosis patients perceive their illness and treatment? Implications for clinical practice. Arch Osteoporos 2012;7:115-24.

39. Kirkham JA, Smith JA, Havsteen-Franklin D. Painting pain: An interpretative phenomenological analysis of representations of living with chronic pain. Health Psychol 2015;34:398-406.

40. Guillemin MM, Westall C. Gaining insight into women's knowing of postnatal depression using drawings. In: Knowing differently: arts-based and collaborative research method. First. Nova Science Publishers; p. 121-39.

41. Witek N, Stebbins GT, Goetz CG. What influences placebo and nocebo responses in Parkinson's disease?: Placebo and Nocebo Response in PD. Mov Disord 2018;33:1204-12. 